\title{
Rursus
}

m. Ruksus

Poiétique, réception et réécriture des textes antiques

$4 \mid 2009$

Erotica

\section{L'expression stylistique du décalage temporel dans Bérénice de Racine}

Résumé de mémoire de stylistique littéraire sous la direction de Madame Lucile Gaudin-Bordes (2007-2008)

Julia Benhamou

\section{CpenEdition}

Journals

Édition électronique

URL : https://journals.openedition.org/rursus/248

DOI : $10.4000 /$ rursus. 248

ISSN : 1951-669X

Éditeur

Université Nice-Sophia Antipolis

Référence électronique

Julia Benhamou, «L'expression stylistique du décalage temporel dans Bérénice de Racine », Rursus [En ligne], 4 | 2009, mis en ligne le 22 janvier 2009, consulté le 21 septembre 2021. URL : http://

journals.openedition.org/rursus/248; DOI : https://doi.org/10.4000/rursus.248

Ce document a été généré automatiquement le 21 septembre 2021

Rursus 


\title{
L'expression stylistique du décalage temporel dans Bérénice de Racine
}

Résumé de mémoire de stylistique littéraire sous la direction de Madame Lucile Gaudin-Bordes (2007-2008)

\author{
Julia Benhamou
}

1 "Tout énoncé s'insère dans le temps et dans une certaine mesure l'exprime ${ }^{1}$ ». Cela s'applique parfaitement à la tragédie classique puisqu'à l'époque classique le temps est constitutif du genre de la tragédie. En effet, la dramaturgie classique est fondée sur des règles impératives dont la plus connue est celle des trois unités d'action, de lieu et de temps. Ces règles convergent vers un seul objectif : la vraisemblance. L'unité de temps contribue à la vraisemblance de l'énoncé. Cependant, par les contraintes qu'elle impose à l'action, l'unité de temps instaure une tension, et elle permet aussi de produire, par le biais du monologue, une parenthèse hors du temps dramatique.

2 Le temps est un ressort dramatique fondamental dans la tragédie et tout particulièrement dans Bérénice. En effet, la pièce fonctionne sur des effets d'attente : un dilemme en amène un autre et le spectateur attend le moment où les personnages se décideront enfin à agir. Le décalage temporel n'est pas exclusif à Bérénice. Ce qui a motivé le choix de Bérénice est l'importance des thèmes relatifs au temps tels que le souvenir, l'urgence du présent, la fuite du temps ou le futur perçu comme une durée interminable. On a reproché à Racine l'absence, dans la pièce, de dénouement fatal. Cependant, Bérénice correspond d'une certaine façon à la conception de la tragédie classique selon laquelle « les héros sont pris dans un conflit avec des forces qui les dépassent mais qui sont très souvent intériorisées ${ }^{2}$ ». Cette intériorisation est atemporelle. Par «atemporel» on entend «qui n'est pas concerné par le temps ». Cette intériorisation fait l'objet d'une étude détaillée à travers le monologue d'Antiochus (acte I, scène 2) et le thème du souvenir et de la douleur. Dans le souvenir, il y a une persistance émotionnelle, ici la douleur, qui entre dans le cadre du « décalage temporel ». Cette persistance entraine le prolongement d'un état d'un moment à un autre. C'est ce déplacement dans le temps que l'on qualifie de « décalage temporel ».

Le décalage temporel peut s'effectuer à travers les tiroirs verbaux, les marqueurs de temps, ou à travers certaines figures de style telles que l'énallage de temps ou bien la 
prolepse, mais aussi par le biais de thèmes tels que le souvenir. En effet, le décalage temporel est constitutif du souvenir. C'est « une impression qui demeure en la mémoire ${ }^{3}$ ». Lorsque le locuteur se remémore ou raconte un souvenir, il déplace un acte ou un état du passé vers le présent en l'amenant à sa mémoire et à celle du destinataire. Ce même souvenir peut engager non seulement le présent mais aussi l'avenir du locuteur qui, en questionnant le présent pose aussi son avenir comme incertain.

4 A travers l'étude du décalage temporel on peut observer que le champ temporel de Bérénice ne se limite pas au présent dramatique mais s'étend bien au-delà grâce aux ressorts du souvenir et de la perception de l'avenir. On constate aussi que chaque personnage a un temps de prédilection et que toutes les orientations temporelles convergent vers le présent dramatique. Antiochus est un personnage orienté vers le passé. Le souvenir est un élément constitutif du personnage et la scène d'exposition s'effectue à partir du récit qu'il fait des évènements qui l'ont conduit à demander un entretien secret avec Bérénice. Dans le monologue d'Antiochus, se produit un va-et-vient entre le passé du locuteur, le présent du « discours-conversation » qu'Antiochus adresse à lui-même ainsi qu'au spectateur, et un avenir présenté comme incertain. Le monologue de genre délibératif, parce qu'il place le personnage dans le dilemme et la réflexion, le place aussi momentanément hors de l'action en créant une parenthèse où le temps ne compte pas. Le thème du souvenir est un ressort dramatique permettant au dramaturge de contourner la règle de l'unité de temps et d'élargir l'action à des faits qui se sont produits en dehors du présent dramatique. Le thème de la douleur associé à celui du souvenir lie passé et présent en établissant une continuité par les émotions. Ainsi, c'est la même émotion, la douleur, persistante, toujours aussi vive que par le passé qui pousse Antiochus à agir. A travers le jeu des temps et tout particulièrement à travers l'emploi du passé composé fonctionnant avec une valeur d'accompli du présent, le passé se révèle comme toujours actuel pour le locuteur. Cela lui donne un caractère tragique, le passé n'étant pour lui qu'un éternel recommencement.

5 En étudiant dans un deuxième temps le récit des évènements historiques et politiques déterminants pour l'enjeu dramatique l'auteur montre que le souvenir peut aussi servir d'argument et donc être lié au présent dramatique. Le récit du siège de Jérusalem par Arsace présente un personnage totalement coupé de son récit, ce que montre ici l'emploi par le locuteur du passé simple constatif. Ici le souvenir ne ravive pas des sentiments, un état, qui durent depuis déjà cinq ans (comme dans la scène 2 de l'acte I), mais il est un argument pour faire l'éloge d'Antiochus, et sert au présent dramatique. Arsace, en tant que confident, ne connaît pas le dilemme ni l'appréhension de l'avenir, ni le regret ou la nostalgie. C'est en effet un personnage ancré dans le présent dramatique. En revanche Bérénice, à travers le récit de l'apothéose de Vespasien et la figure de l'hypotypose, montre un regard tourné vers le passé et une certaine appréhension de l'avenir. La nonvérité dans le présent dramatique de l'argumentation de Bérénice, égarée par un " souvenir charmant ", indique un décalage temporel. Il correspond à une vérité révolue, et contenue dans l'hypotypose, confrontée à la réalité du présent dramatique. Dans la tirade d'Antiochus (acte I, scène 4), le décalage temporel se produit dans l'alternance du récit de la passion d'Antiochus et des commentaires qu'il fait de ce récit et qui le mettent à jour au regard du présent dramatique. En effet, les commentaires du locuteur créent, à première vue, une rupture avec le temps du récit, mais par un jeu des temps et par l'absence parfois de tiroir verbal qui sont le reflet des mouvements de la conscience du 
locuteur, les commentaires permettent de donner au récit une portée dans le présent dramatique.

Dans la seconde partie est étudié le décalage temporel présent/futur à travers le personnage de Titus. C'est en effet un personnage orienté vers l'avenir, le seul à percevoir la notion de «temps perdu» qu'il manifeste par le biais du lyrisme dans le monologue de la scène 4 de l'acte IV. La notion de fuite du temps correspond à différents réglages énonciatifs et plus particulièrement à l'emploi du présent élargi et du passé composé à valeur d'accompli qui donnent une sensation de ralentissement du temps, mais aussi le sentiment d'un temps à rattraper qui se manifeste par l'accélération des derniers vers du monologue.

7 L'énallage temporelle, qui reste une option de lecture, fait partie des procédés qui permettent à la langue de la tragédie classique, rendue artificielle par les vers, d'acquérir un certain naturel propre au langage parlé. De plus, elle apporte une profondeur à la fois temporelle, aspectuelle et modale qui dépasse la valeur de base du présent en lui apportant celle du temps ou du mode auquel il se substitue. Dans Bérénice, on relève quelques occurrences d'énallage de temps. Au présent des verbes commentés on pourrait substituer le futur. C'est une forme de décalage temporel en cela que le locuteur avance un évènement dont la réalisation est considérée comme inévitable. De même l'étude du tétramètre permet de constater que, par le biais de ce rythme, certains faits ne sont plus présentés comme une succession logique mais sont soit placés hors du temps, soit présentés comme une fatalité. Ce caractère d'inéluctabilité produit une impression d'accélération du temps, voire de fuite du temps.

Enfin, dans l'évocation de Rome par Titus et Paulin, le décalage temporel exprime un décalage de point de vue. Il se manifeste par un emploi des temps différent dans les discours de Titus et de Paulin. Paulin évoque Rome dans une continuité, comme si elle était l'incarnation d'une tradition, par l'emploi du présent de l'indicatif. En revanche le regard de Titus est tourné vers l'avenir, tout d'abord sujet au doute, puis catégorique comme en témoigne l'emploi du présent et du futur. Toute projection dans l'avenir n'a de sens dans la tragédie que lorsqu'on peut déjà sentir ses conséquences directes sur le présent dramatique. Ainsi que ce soit par le décalage passé/présent, présent/passé ou présent/futur, tous les éléments de l'action convergent vers le présent dramatique.

A travers l'étude du décalage temporel dans Bérénice, l'auteur a voulu décrire un des moyens utilisés par le dramaturge pour contourner la règle des trois unités. Il se base, dans Bérénice, sur le décalage temporel. En effet, en contournant la règle de l'unité de temps, c'est-à-dire en projetant le récit, lors de brefs épisodes, dans une autre époque que celle du présent dramatique tout en reliant ce même récit au présent dramatique par certains procédés, le dramaturge peut aussi intégrer des actions secondaires telles que le siège de Jérusalem ou bien la rencontre de Titus et Bérénice, et il peut bien évidemment les transposer dans un autre lieu que celui de l'action principale : l'auteur fait ainsi de la contrainte de l'unité de temps un atout. 


\section{NOTES}

1. LARTHOMAS Pierre, Notions de stylistique générale, 1998, Presses universitaires de France, Paris, p. 83.

2. JARRETY Michel, Lexique des termes littéraires, 2001, Librairie Générale Française, Paris, p. 450.

3. LITTRE ET BEAUJEAN, Dictionnaire de la langue française, Hachette, Paris, p. 1130.

\section{AUTEUR}

\section{JULIA BENHAMOU}

Etudiante de Mastère (Lettres classiques, Linguistique) 